typeset using JPSJ.sty $<$ ver.1.0b $>$

\title{
Correlation Effects on Optical Conductivity of FeSi
}

\author{
Kentaro URASAKI and Tetsuro SASo** \\ Department of Physics, Saitama University, Urawa, 338-8570
}

(Received June 19, 2018)

\begin{abstract}
Effects of electron correlation in FeSi are studied in terms of the two-band Hubbard model with the density of states obtained from the band calculation. Using the self-consistent secondorder perturbation theory combined with the local approximation, the correlation effects are investigated on the density of states and the optical conductivity spectrum, which are found to reproduce the experiments done by Damascelli et al. semiquantitatively. It is also found that the peak at the gap edge shifts to lower energy region by correlation effects, as is seen in the experiments.
\end{abstract}

KEYWORDS: FeSi, optical conductivity, Kondo insulator, two-band Hubbard model

FeSi is a well-known material for its anomalous properties that cannot be understood from conventional band theories. At low temperatures FeSi is a nonmagnetic semiconductor with a gap size of about $50 \mathrm{meV}$, whereas it has a magnetic moment and shows metallic behaviors at room temperatures 因, To explain the magnetic properties, Takahashi and Moriya ${ }^{\text {B }}$ ) applied the spin fluctuation theory leading to an idea of thermally induced local moment, which was confirmed

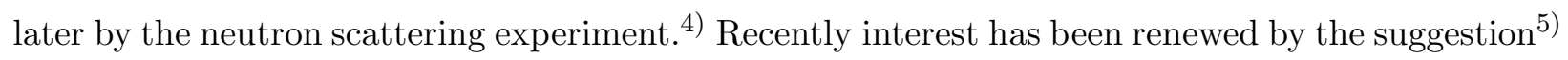
that FeSi can be viewed as a strongly correlated insulator or a Kondo insulator. Kondo insulators have been found in the $f$-electron systems and typical examples are $\mathrm{YbB}_{12}$, (,) $\mathrm{Ce}_{3} \mathrm{Bi}_{4} \mathrm{Pt}_{3} \mathrm{O}$ ) and so on. They have correlated $f$-bands and become insulators with small energy gaps at low temperatures. The correlation in FeSi may not be so strong as in these materials. It will be, however, of interest to reinvestigate the properties of FeSi from a new point of view.

Low temperature properties of FeSi have been studied intensively from this aspect. Especially, Schlesinger et al. 1 ) measured the optical conductivity and reported that the gap $(\sim 50 \mathrm{meV})$ at low temperatures disappears at a temperature $(\sim 250 \mathrm{~K})$ lower than the gap size, which they attributed to the correlation effect. The optical conductivity of FeSi was reported by several groups. 8.9, (1), (10) The gap opening of about $50 \mathrm{meV}$ and the rapid temperature variation were commonly observed.

\footnotetext{
* e-mail address: kentaro@krishna.th.phy.saitama-u.ac.jp

** e-mail address: saso@phy.saitama-u.ac.jp
} 
Those optical measurements also provided us with a direct information about the dynamical structure of this system at low temperatures such as a precise gap size. Moreover, high-resolution photoemission measurement was done by Saitoh et al.,12) who estimated the self-energy of the $d$-electrons and demonstrated a possible existence of the strong renormalization of the electronic states at low energies. A recent experiment reported that a sign of the correlation effects is found in the tunneling spectroscopy measurements, 13) where the gap in the density of states (DOS) seems to be strongly temperature dependent. It was also reported that the low temperature transport measurements for $\mathrm{Fe}_{1-x} \mathrm{Co}_{x} \mathrm{Si}(14)$ and $\mathrm{FeSi}_{1-x} \mathrm{Al}_{x} 15$ suggest strong mass enhancement of carriers.

In these contexts several theoretical attempts have been made to take the correlation effects into account. Among these attempts Fu and Doniach 1 ( $)$ proposed a two-band Hubbard model based on their band calculation.17) Their calculation revealed the importance of the correlation effects on physical quantities but there seems to be some errors about the treatment of the self-energies. Using this model, we 18) also calculated physical quantities correctly, and showed that the temperature variations of the magnetic susceptibility and the optical conductivity are consistent with the experiments. From a viewpoint of the strongly correlated systems, Riseborough $\$$ ) calculated the angle-resolved-photoemission spectrum of the Anderson lattice model in terms of the $1 / N$ expansion.

On the other hand several band calculations have been done for FeSi.24, 27, 22, 23,21] Each of them succeeded in giving the insulating ground state and showed that the minimum gap is given by the indirect one, but it is close to a direct one. It has been also demonstrated that the states near the Fermi level mainly consist of the $d$-electrons. However, somewhat different gap sizes $(0.03-0.2 \mathrm{eV})$ were reported. There are some attempts to explain the physical properties of FeSi by extending the band calculations, e.g., Jarlborg24) investigated the thermal disorder effect and Anisimov et al.25) applied the LDA $+U$ scheme. Fu et al.47) calculated the optical conductivity without the correlation effect using their band calculation. As a result, the necessity of the correlation effects was underlined in order to reproduce the observed temperature variation. Ohta et al 9 also calculated the optical conductivity in the joint-DOS form from their band calculation and showed that the gap is filled with increasing temperature. In their calculation, the Drude contribution is estimated from the experimental DC resistivity and is added to the joint-DOS term separately. However, the flat part of the optical conductivity spectrum within the gap is not reproduced.

Recently, Yamada et al.26) calculated the DOS within the LMTO-ASA method carefully, and obtained the gap size close to the experimental one. Therefore, in the present Letter, we study the two-band Hubbard model using the Fe part of the DOS obtained from this band calculation and calculate the temperature variation of the optical conductivity to investigate the correlation effects. Moreover, we compare the calculated spectrum of the optical conductivity with the experimental data and estimate the gap size (indirect one) in the DOS of the real system. 
We start from the following two-band Hubbard Hamiltonian:

$$
\begin{aligned}
H= & \sum_{i j \sigma}\left(t_{i j}^{1} c_{i 1 \sigma}^{\dagger} c_{j 1 \sigma}+t_{i j}^{2} c_{2 i \sigma}^{\dagger} c_{2 j \sigma}\right) \\
& +U \sum_{i}\left(n_{i 1 \uparrow} n_{i 1 \downarrow}+n_{i 2 \uparrow} n_{i 2 \downarrow}\right) \\
& +U_{2} \sum_{i}\left(n_{i 1 \uparrow} n_{i 2 \downarrow}+n_{i 2 \uparrow} n_{i 1 \downarrow}\right) \\
& +U_{3} \sum_{i}\left(n_{i 1 \uparrow} n_{i 2 \uparrow}+n_{i 2 \downarrow} n_{i 1 \downarrow}\right) \\
& -J \sum_{i}\left(c_{i 1 \uparrow}^{\dagger} c_{i 1 \downarrow} c_{i 2 \downarrow}^{\dagger} c_{i 2 \uparrow}+c_{i 2 \uparrow}^{\dagger} c_{i 2 \downarrow} c_{i 1 \downarrow}^{\dagger} c_{i 1 \uparrow}\right),
\end{aligned}
$$

where the $c_{i a \sigma}^{\dagger}\left(c_{i a \sigma}\right)$ creates (destroys) an electron on site $i$ in band $a=1,2$ with spin $\sigma$. If the two bands are degenerate, this Hamiltonian is rotationally invariant in spin and real spaces when one chooses $U_{2}=U-J$ and $U_{3}=U-2 J$ where $U$ and $J$ denotes the Coulomb repulsion and the exchange interaction, respectively. We adopt this relation so as to reduce the number of parameters.

The initial DOS obtained from the band calculation 26 ) is displayed in Fig. 1 (see the solid line for $T=0$ ), where we widen the energy gap by $16 \%$ so as to reproduce the gap edge of the optical conductivity at $4 \mathrm{~K}$ of the experiment. There is an ambiguity to determine the gap size $E_{g}$. If we extrapolate the steepest part of the DOS at the both sides of the gap neglecting the tails, we obtain $E_{g}=75 \mathrm{meV}$, whereas if we regard the gap as the region inside the tails of the gap edge, we obtain $60 \mathrm{meV}$. The Fermi level $\left(E_{F}=0\right)$ is placed at the center of the gap at $T=0$, and we call the upper and the lower part of the DOS as the band 1 and 2, respectively. We introduce a cut off for each band so as to include one state per spin in each band. Then the band width for the band 1 is about $0.56 \mathrm{eV}$ and about $0.63 \mathrm{eV}$ for the band 2. Moreover, although the DOS is asymmetric, we assume that the chemical potential is temperature independent. This means that the electron number of this system varies with temperature. This assumption seems to be reasonable in the real system, where electrons and holes are supplied from other bands.

Because the present interests are on the correlation effects in the low energy and low temperature region of this model, we adopt the self-consistent second-order perturbation theory (SCSOPT) combined with the local approximation.28) In SCSOPT, the self-energies $\Sigma_{1}^{(2) \sigma}(\omega)$ and $\Sigma_{2}^{(2) \sigma}(\omega)$ are calculated as

$$
\begin{aligned}
\Sigma_{1}^{(2) \sigma}(\omega)= & \iiint_{-\infty}^{\infty} d \varepsilon_{1} d \varepsilon_{2} d \varepsilon_{3} \\
& {\left[U^{2} \rho_{1}^{-\sigma}\left(\varepsilon_{1}\right) \rho_{1}^{\sigma}\left(\varepsilon_{2}\right) \rho_{1}^{-\sigma}\left(\varepsilon_{3}\right)\right.} \\
& +U_{2}^{2} \rho_{2}^{-\sigma}\left(\varepsilon_{1}\right) \rho_{1}^{\sigma}\left(\varepsilon_{2}\right) \rho_{2}^{-\sigma}\left(\varepsilon_{3}\right)
\end{aligned}
$$




$$
\begin{gathered}
+U_{3}^{2} \rho_{2}^{\sigma}\left(\varepsilon_{1}\right) \rho_{1}^{\sigma}\left(\varepsilon_{2}\right) \rho_{2}^{\sigma}\left(\varepsilon_{3}\right) \\
\left.+J^{2} \rho_{2}^{-\sigma}\left(\varepsilon_{1}\right) \rho_{2}^{\sigma}\left(\varepsilon_{2}\right) \rho_{1}^{-\sigma}\left(\varepsilon_{3}\right)\right] \\
\times \frac{f\left(-\varepsilon_{1}\right) f\left(\varepsilon_{2}\right) f\left(\varepsilon_{3}\right)+f\left(\varepsilon_{1}\right) f\left(-\varepsilon_{2}\right) f\left(-\varepsilon_{3}\right)}{\omega+\varepsilon_{1}-\varepsilon_{2}-\varepsilon_{3}+\mathrm{i} \delta}, \\
\Sigma_{2}^{(2) \sigma}(\omega)=(1 \leftrightarrow 2),
\end{gathered}
$$

where

$$
\rho_{a}^{\sigma}(\omega)=-\frac{1}{\pi} \operatorname{Im} G_{a}^{\sigma}(\omega+\mathrm{i} \delta)
$$

and

$$
\begin{aligned}
G_{a}^{\sigma}(\omega) & =\frac{1}{N} \sum_{\mathbf{k}} G_{a}^{\sigma}(\mathbf{k}, \omega) \\
& =\int_{-\infty}^{\infty} d \varepsilon \rho_{a}^{0 \sigma}(\varepsilon) \frac{1}{\omega-\varepsilon-\Sigma_{a}^{(2) \sigma}(\omega)} .
\end{aligned}
$$

Here, $N$ is the number of sites, $f(\varepsilon)$ the Fermi function and $\rho_{a}^{0 \sigma}(\varepsilon)$ the DOS of band $a$ for the non-interacting case. We solve the set of equations (2)-(4) self-consistently. To make numerical calculation easy, we take $\delta$ finite $\left(\delta=10^{-4}\right)$ in eq. (3) and convert these equations with the transformations 28 .

$$
\begin{aligned}
& A_{a}^{\sigma}(\tau)=\int_{-\infty}^{\infty} d \epsilon e^{-\mathrm{i} \tau \varepsilon} \rho_{a}^{\sigma}(\varepsilon) f(\varepsilon), \\
& B_{a}^{\sigma}(\tau)=\int_{-\infty}^{\infty} d \epsilon e^{-\mathrm{i} \tau \varepsilon} \rho_{a}^{\sigma}(\varepsilon) f(-\varepsilon) .
\end{aligned}
$$

In general, the current operator is expressed as

$$
j=e \sum_{\sigma, \mathbf{k}} \sum_{m m^{\prime}} v_{\mathbf{k}}^{m m^{\prime}} c_{m \mathbf{k}}^{\dagger} c_{m^{\prime} \mathbf{k}}
$$

where $m$ denotes the band index. To derive the expression for the optical conductivity, we assume the intra- and interband contribution to be equal $\left(v_{\mathbf{k}}^{m m^{\prime}}=v_{\mathbf{k}}\right)$. Using the linear response theory, we assume that the momentum conservation is violated in real systems by defects and phonon-assisted transitions and replace $v_{\mathbf{k}} v_{\mathbf{k}^{\prime}} \delta_{\mathbf{k k}^{\prime}}$ with $v^{2} / N$ in the current-current correlation function. Then, we finally obtain a joint-DOS-like form for the optical conductivity:

$$
\begin{array}{r}
\sigma(\omega, T)=\frac{\pi(e v)^{2}}{\hbar} \sum_{\sigma} \int_{-\infty}^{\infty} d \nu \frac{f(\nu)-f(\nu+\omega)}{\omega} \\
\times\left[\rho_{1}^{\sigma}(\nu)+\rho_{2}^{\sigma}(\nu)\right]\left[\rho_{1}^{\sigma}(\nu+\omega)+\rho_{2}^{\sigma}(\nu+\omega)\right] .
\end{array}
$$

In the numerical calculation we set $(e v)^{2} / \hbar=1$ for simplicity.

Figure 1 shows the temperature dependence of the quasiparticle DOS for $U=0.5 \mathrm{eV}$ and $J=$ $0.35 U$. These values are chosen so as to reproduce the shape and the temperature dependence of 


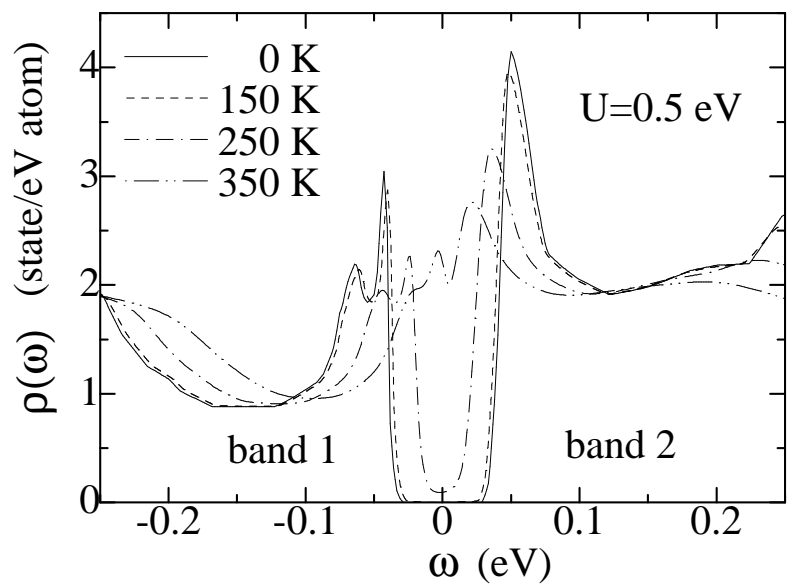

Fig. 1. The initial DOS obtained from the band calculation(Ref. 26) at $T=0$. At finite $T$, the DOS is strongly temperature dependent due to the correlation effects.

the optical conductivity spectrum. At $0 \mathrm{~K}$, the band 1 is filled and the band 2 is empty, so that the correlation effect is absent except the Hartree-Fock contribution. At finite $T$, however, the correlation is introduced through the thermally excited electrons and holes. As a result, the DOS becomes strongly temperature dependent and is smeared especially near the gap. The gap at $0 \mathrm{~K}$ is almost filled up at the temperature of the order of its size and it is also found that the peaks of the gap edges move toward the center of the gap.

In Fig. 2(a), the temperature variation of the optical conductivity calculated from eq. (7) is shown. Since the intraband (Drude) contribution does not exist at $0 \mathrm{~K}$, only the interband contribution survives and seems to reproduce the shape of the spectrum of the experiment at $4 \mathrm{~K}$. The slightly rounded gap edge in the calculation is due to the tails at the gap edge in the DOS of the band calculation.26) If we extrapolate the steepest parts of the edge, we obtain the gap size as about 75 $\mathrm{meV}$, which is consistent with the gap size of the DOS. Since these features reflect the shape of the DOS directly (see eq.(7)), the band calculation using LMTO-ASA by Yamada et al.26) seems to give good results about the whole structure of the DOS at $T=0$ except for a slightly smaller gap size.

At finite temperatures, the experiment done by Damascelli et al.11) (Fig. 2(b)) shows not only that the gap is almost filled up at $300 \mathrm{~K}$, but that the increase of the weight in the gap from 100 to $300 \mathrm{~K}$ seems to be more rapid than that from 4 to $100 \mathrm{~K}$. One can also recognize that the peak at the gap edge shifts to the lower frequency region as the temperature rises. In our calculation (Fig. $2(\mathrm{a})$ ), the gap is almost filled up at $300 \mathrm{~K}$ as well as the rapid increase from 150 to $300 \mathrm{~K}$, but a little slower compared to the experiment, which may be mainly because the absence of the correlation 


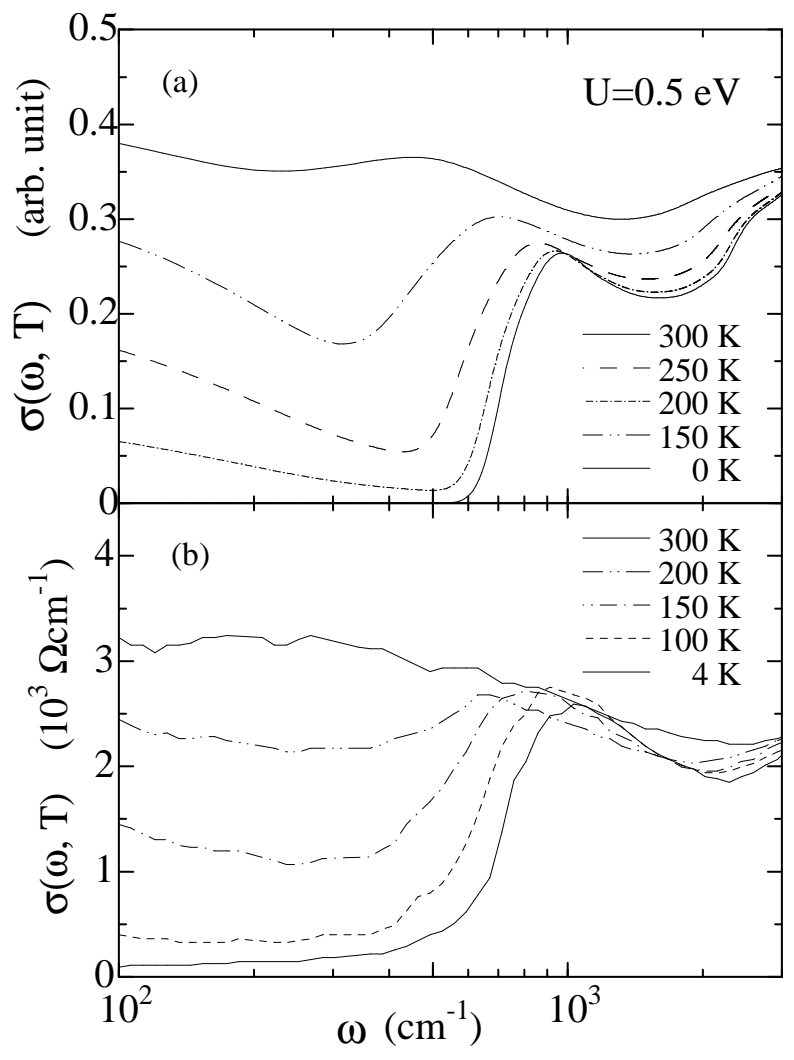

Fig. 2. (a)The temperature dependence of the optical conductivity calculated with the eq. (7). (b)The experimental data from Ref. 11. The peaks due to phonons observed in the gap are omitted.

effect at $0 \mathrm{~K}$ in the present model. Reflecting the correlation effects the peak at the gap edge shifts to lower frequency region, as is seen in the experiment. In our calculation, however, there are dips between the Drude and the interband contributions in contrast to the experiment. This may be caused by the assumption in the previous formulation, where we neglected the $\mathbf{k}$-dependence of the current operator and took the intraband (Drude) and interband contribution to be equal in deriving eq. (7).

For comparison, the optical conductivity obtained from the Hartree-Fock approximation or a rigid band model is shown in Fig. 3. Since the gap is filled only with the Drude contribution, the temperature variation is monotonous and the gap is not efficiently filled at a temperature of the order of the gap size as pointed out by Fu et al. 1 ) The peak at the gap edge does not shift. These results are consistent with the previous studies, 17.6) but are in disagreement with the experiments.

In summary, we have calculated the correlation effects using the two-band Hubbard model and the DOS obtained from the band calculation, and compared the calculated optical conductivity with the experiment. Although we used an essential but simplified model and applied the local 


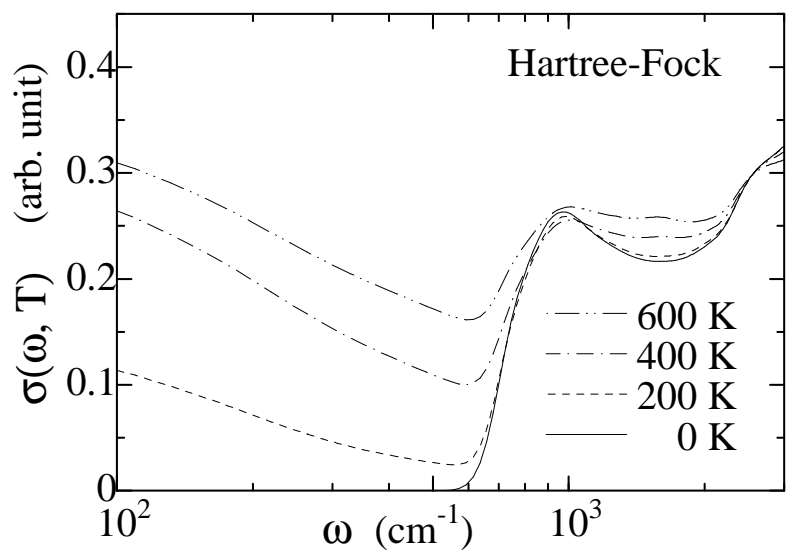

Fig. 3. Calculated optical conductivity within the Hartree-Fock approximation.

approximations in deriving eq. (7), the calculated optical conductivity reproduced the experiment rather well. Thus we determined the gap size (indirect one) as $75 \mathrm{meV}$ when the tail is neglected (60 meV within the tail). Furthermore, we confirmed that the correlation effect is essential to explain the rapid temperature dependence of the optical conductivity, and gave a direct explanation for the shift of the peak at the gap edge. The latter is another possible sign of the correlation effects. Similar effect is also seen in the optical measurement of $\mathrm{YbB}_{12}, 30$ ) but the shift is observed on the IR peak instead of the peak at the minimum gap edge.

In the present model the higher order terms of the electron correlation effect are quenched at $T=0$. The renomalization effects at $T=0$, however, may be also important as in the model by $\mathrm{Fu}$ and Doniach 16) The optical conductivity of this model is strongly temperature dependent as is seen in our previous calculation. 18 It is also important to take the spin fluctuations 3 , 31) into account at higher temperatures or for magnetic properties. Furthermore, to incorporate the details of the band dispersions into the methods of the strongly correlated electron systems is our present interest.

\section{Acknowledgements}

The authors would like to thank Professor H. Yamada for providing them the details of the band calculation (LMTO-ASA) for FeSi and for his useful comments. This work is supported by Grant-in-Aid for Scientific Research No.11640367 from the Ministry of Education, Science, Sports and Culture.

[1] V. Jaccarino, G. K. Wertheim, J. H. Wernick, L. R. Walker and S. Arajs: Phy. Rev. 160 (1967) 476.

[2] H. Watanabe, H. Yamamoto and K. Ito: J. Phys. Soc. Jpn. 18 (1963) 995. 
[3] Y. Takahashi and T. Moriya: J. Phys. Soc. Jpn. 46 (1979) 1451;Y. Takahashi, J. Phys.: Cond. Matter 9 (1997) 2593.

[4] G. Shirane, J. E. Fisher, Y. Endoh and K. Tajima: Phys. Rev. Lett. 59 (1987) 351; K. Tajima, Y. Endoh, J. E. Fisher and G. Shirane: Phys. Rev. B 38 (1988) 6954.

[5] G. Aeppli and Z. Fisk: Comments Condens. Matter Phys. 16 (1992) 155.

[6] M. Kasaya: J. Mag. Magn. Mater. 47 \& 48 (1985) 429.

[7] M. F. Hundley, P. C. Canfield, J. D. Thompson, Z. Fisk and J. M. Laurence: Phys. Rev. B 42 (1990) 4862.

[8] Z. Schlesinger, Z. Fisk, Hai-Tao Zhang, M. B. Maple, J. F. DiTusa and G. Aeppli: Phys. Rev. Lett. 71 (1993) 1748 .

[9] H. Ohta, S. Kimura, E. Kulatov, S. V. Halilov, T. Nanba, M. Motokawa, M. Sato and K. Nagasawa: J. Phys. Soc. Jpn. 63 (1994) 4206.

[10] S. Paschen, E. Felder, M. A. Chernikov, L. Degiorgi, H. Schwer, H. R. Ott, D. P. Young, J. L. Sarrao and Z. Fisk: Phys. Rev. B 56 (1997) 12916.

[11] A. Damascelli, K. Schulte, D. van der Marel, M. Fäth and A. A. Menovsky: Physica B 230-232 (1997) 787.

[12] T. Saitoh, A. Sekiyama, T. Mizokawa, A. Fujimori, K. Ito, H. Nakayama and M. Shiga: Solid State Commun. 95 (1995) 307.

[13] M. Fäth, J. Aarts, A. A. Menovsky, G. J. Nieuwenhuys and J. A. Mydosh: Phys. Rev. B 58 (1995) 15483.

[14] M. A. Chernikov, L. Degiorgi, E. Felder, S. Paschen, A. D. Bianchi, H. R. Ott, J. L. Sarrao, Z. Fisk and D. Mandrus: Phys. Rev. B 56 (1997) 1366.

[15] J. F. DiTusa, K. Friemelt, E. Bucher, G. Aeppli and A. P. Ramirez: Phys. Rev. B 58 (1998) 10288.

[16] C. Fu and S. Doniach: Phys. Rev. B 51 (1995) 17439.

[17] C. Fu, M. P. C. M. Krijn and S. Doniach: Phys. Rev. B 49 (1994) 2219.

[18] K. Urasaki and T. Saso: Phys. Rev. B 58 (1998) 15528.

[19] P. S. Riseborough: Phys. Rev. B 58 (1998) 15534.

[20] L. F. Mattheiss and D. R. Hamann: Phys. Rev. B 47 (1993) 13114.

[21] E. Kulatov and H. Ohta: J. Phys. Soc. Jpn. 66 (1997) 2386.

[22] T. Jarlborg: Phys. Rev. B 51 (1995) 11106.

[23] V. R. Galakhov, E. Z. Kurmaev, V. M. Cherkashenko, Yu M. Yarmoshenko, S. N. Shamin, A. V. Postnikov, St Uhlenbrock, M. Neumann, Z. W. Lu, B. M. Klein and Zhu-Pei Shi: J. Phys.: Condens. Matter 7 (1995) 5529.

[24] T. Jarborg: Phys. Rev. B 59 (1999) 15002.

[25] V. I. Anisimov, S. Yu Ezhov, I. S. Elfimov and I. V. Solovyev: Phys. Rev. Lett. 76 (1996) 1735.

[26] H. Yamada, K. Terao, H. Ohta, T.Arioka and E. Kulatov: J. Phys.: Condens. Matter 11 (1999) L309.

[27] R. H. Parmenter: Phys. Rev. B 8 (1973) 1273.

[28] E. Müller-Hartmann: Z. Phys. 76211 (1989).

[29] A. Georges, G. Kotliar, W. Krauth and M. J. Rozenberg: Rev. Mod. Phys. 68 (1996) 13.

[30] H. Okamura, S. Kimura, H. Shinozaki, T. Nanba, F. Iga, N. Shimizu and T. Takabatake: Phys. Rev. B 58 (1998) R7496.

[31] T. Saso, submitted to J. Phys. Soc. Jpn. 


\section{Figure Captions}

Figure 1: The initial DOS obtained from the band calculation(Ref. 26) at $T=0$. At finite $T$, the

DOS is strongly temperature dependent due to the correlation effects.

Figure 2: (a)The temperature dependence of the optical conductivity calculated with the eq. (7).

(b)The experimental data from Ref. 11. The peaks due to phonons observed in the gap are omitted.

Figure 3: Calculated optical conductivity within the Hartree-Fock approximation. 